\title{
ANÁLISES COPROPARASITOLÓGICAS DE AVES SILVESTRES CATIVAS
}

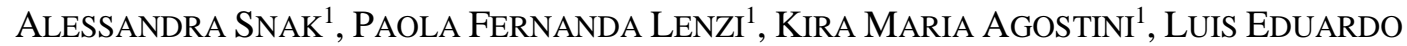 \\ DELGADO $^{2}$, ClEUZA Rocha MONTANUCCI ${ }^{3}$, MARIVONE VALENTIM ZABOTT ${ }^{4}$
}

\author{
${ }^{1}$ Graduada em Medicina Veterinária pela Universidade Federal do Paraná, Palotina, PR, Brasil- \\ alessandra.snak@hotmail.com \\ ${ }^{2}$ Médico Veterinário da Prefeitura Municipal de Cascavel, Fundação para o Desenvolvimento \\ Científico e Tecnológico, Cascavel, PR, Brasil \\ ${ }^{3}$ Técnica da Universidade Federal do Paraná, Palotina, PR, Brasil \\ ${ }^{4}$ Professora Doutora da Universidade Federal do Paraná, Palotina, PR, Brasil
}

\begin{abstract}
Na maioria dos casos de parasitismo em aves cativas não há expressão de sinais clínicos. Esse fato aponta a importância da realização de exames parasitológicos frequentes nesses animais. $\mathrm{O}$ objetivo do presente trabalho foi realizar exames coproparasitológicos para monitorar as infecções parasitárias nas aves cativas no Parque Municipal Danilo Galafassi (Zoológico Municipal de Cascavel-PR), projeto aprovado pela Comissão de Ética e Bem-estar Animal (CEBEA) do Campus Palotina- UFPR, protocolo $n^{\circ}$ 29/2010. Foram analisadas 228 amostras de fezes de 37 espécies de aves cativas, provenientes de 22 recintos, no período de agosto de 2010 a julho de 2012. As fezes refrigeradas foram analisadas por meio dos métodos de flutuação de Willis-Mollay e de sedimentação simples
\end{abstract}

\section{RESUMO}

de Hoffmann, Pons e Janer. Das amostras analisadas, 127 $(55,7 \%)$ foram positivas e, destas, 55 (43,3\%) apresentaram infecção mista. Os parasitos encontrados pertencem aos gêneros Strongyloides, Eimeria, Capillaria, Deletrocephalus e Isospora, às superfamílias Strongyloidea, Ascaroidea e Spiruroidea, à ordem Trichurida e à classe Cestoda. Os resultados dos exames dos recintos de Jabiru mycteia (tuiuiú) e Cariama cristata (seriema) foram negativos, em todo o período avaliado. Medidas higiênico-sanitárias e de controle da circulação de animais e pessoas foram instituídas para minimizar a infecção das aves, porém são necessárias novas análises para avaliação do impacto das mesmas.

PALAVRAS-CHAVE: aves cativas; diagnóstico parasitológico; zoológico.

\section{COPROPARASITOLOGICAL ANALYSIS OF CAPTIVE WILD BIRDS}

\section{ABSTRACT}

In most instances of parasitism in captive birds there is no expression of clinical signs. This fact underscores the importance of performing frequent parasitological examinations in these animals. The aim of this study was to do a coproparasitological analysis to monitor parasitic infections in captive birds in Danilo Galafassi Municipal
Park (Municipal Zoo of Cascavel-PR), project approved by the Committee of Ethics and Animal Welfare (CEBEA) Campus Palotina-UFPR, protocol no 29/2010. A total of 228 fecal samples were analyzed from 37 species of captive birds from 22 enclosures in the period from August 2010 to July 2012. Chilled feces were analyzed 
through flotation methods of Willis-Mollay and simple sedimentation of Hoffmann, Pons and Janer. Out of the analyzed samples, $127(55.7 \%)$ were positive and among these $55(43.3 \%)$ presented mixed infection. The parasites that were found belonged to the genus Strongyloides, Eimeria, Capillaria, Deletrocephalus and Isospora, the superfamilies Strongyloidea, Ascaroidea and Spiruroidea,

KEYWORDS: : captive birds; parasitological diagnosis; zoo.

\section{INTRODUÇÃO}

As doenças parasitárias costumam ser mais frequentes e causar quadros mais graves em animais que vivem em cativeiro, quando comparados àqueles de vida livre. Isso se deve a diversos fatores como: estresse, higiene, nutrição e área restrita, contribuindo para que determinadas espécies de parasitos possam concluir seus ciclos, causando infecções $^{1}$. O parasitismo por endoparasitos é comum em aves mantidas em cativeiro. Esses agentes podem causar infecções e doenças de acordo com o tipo de manejo, resistência dos animais, potencial biótico dos patógenos e a própria rusticidade destas aves. Vale ressaltar que as aves silvestres são suscetíveis a diversas doenças comumente diagnosticadas nas aves domésticas ${ }^{2}$.

A precisão do diagnóstico de doenças parasitárias depende de vários fatores tais como a colheita correta das amostras, forma de conservação, armazenamento e transporte ao laboratório e os métodos de diagnóstico laboratoriais utilizados. Além disso, o tempo de armazenamento das amostras de fezes pode interferir no diagnóstico, uma liberando as larvas ${ }^{3}$.

Os Jardins Zoológicos, particularmente se localizados em uma grande cidade, representam um "ecossistema artificial", com sua variedade de espécies de animais silvestres e exóticos e vivendo em condições diferentes de seu habitat natural. Além disso, esse ecossistema é geralmente frequentado por animais domésticos e sinantrópicos, provenientes do vez que os ovos de diversos parasitos podem eclodir the order Trichurida and the class Cestoda. The examinations results of the enclosure of Jabiru mycteia (tuiuiú) and Cariama cristata (seriema) were negative throughout the study period. Hygienic-sanitary measures and movement control measures of animals and people circulation were introduced to minimize bird's infection, but it requires further analysis to assess their impact. meio urbano e suburbano circunvizinho, tais como pombos, pardais e outras aves, camundongos, ratos e demais roedores gambás e gatos errantes ${ }^{4}$.

A importância de estudos na área de parasitologia de animais silvestres se deve ao fato de serem hospedeiros e reservatórios, podendo influenciar na saúde dos ecossistemas e dos ambientes naturais e domésticos 5 .

O Parque Municipal Danilo Galafassi, conhecido como Zoológico Municipal de Cascavel, possui uma área de 17,91 ha, sendo parte integrante do Parque Municipal Paulo Gorski. Atualmente conta com cerca de 340 animais de 68 espécies, sendo 37 de aves, 23 de mamíferos e oito de répteis. Os animais foram adquiridos através de permutas com outras instituições, doações supervisionadas pelo IBAMA, nascimentos no próprio zoológico e apreensão de animais realizada pelo IBAMA e Polícia Florestal ${ }^{6}$. O objetivo do presente trabalho foi realizar exames coproparasitológicos para monitorar as infecções parasitárias nas aves cativas no Parque Municipal Danilo Galafassi, em CascavelPR.

\section{MATERIAL E MÉTODOS}

Foram realizadas coletas trimestrais, no período de agosto de 2010 a julho de 2012, totalizando 228 amostras de fezes de 37 espécies de aves cativas, em 22 recintos do Parque Municipal Danilo Galafassi (Figura 1). 


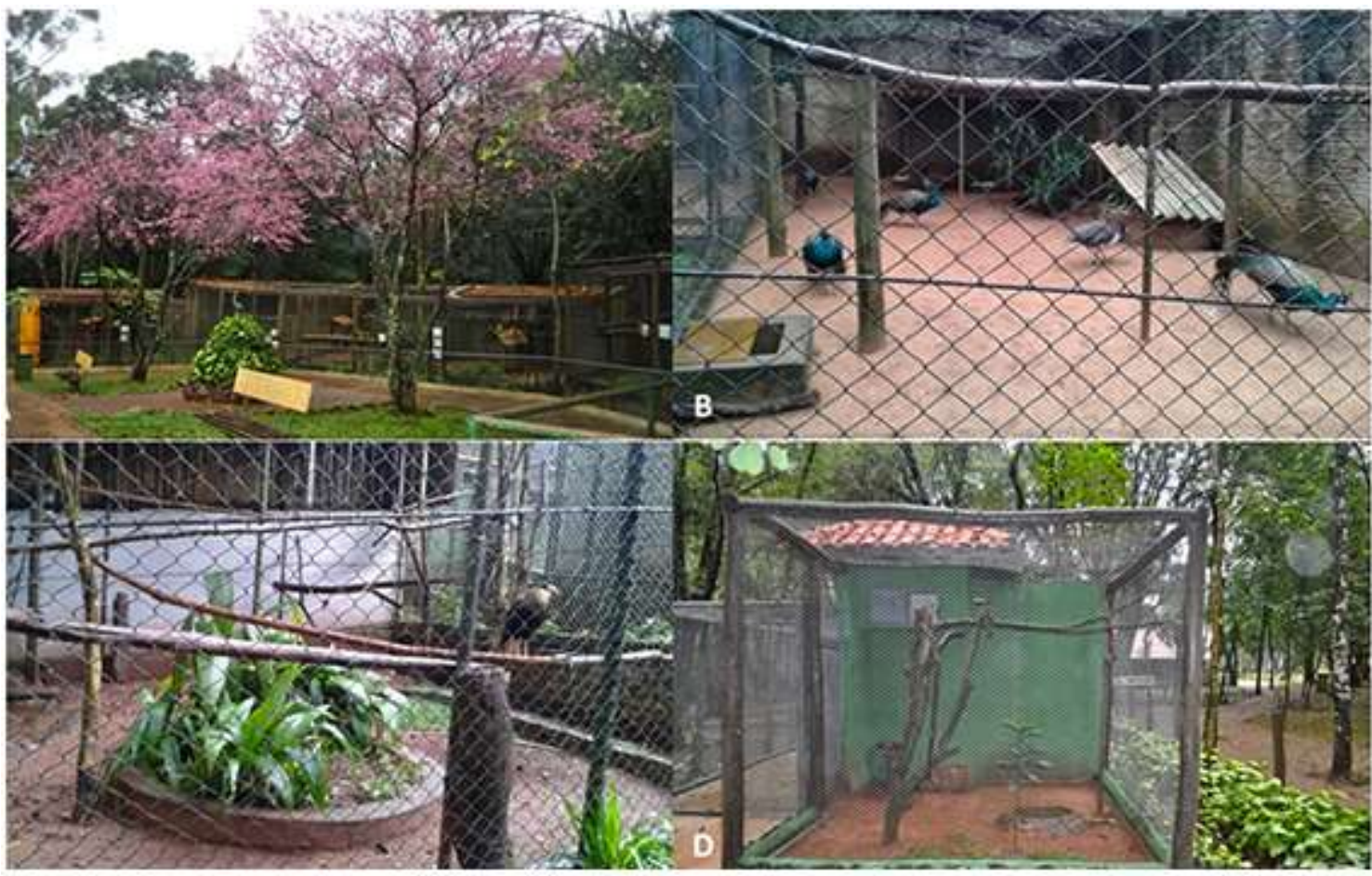

Figura 1 - (A) ambiente dos recintos das aves do Parque Municipal Danilo Galafassi; (B) recinto dos pavões (Pavo cristatus); (C) recinto dos Carcarás (Polyborus plancus); (D) recinto dos araçaris castanhos (Pteroglossus castanotis).

As fezes foram coletadas do chão destes recintos, sendo selecionadas aquelas com aparência fresca e com poucas sujidades. As amostras foram acondicionadas sem conservantes em frascos limpos com tampa de rosca e refrigeradas até posterior análise, em até sete dias. As análises foram realizadas no Laboratório de Parasitologia e Doenças Parasitárias da Universidade Federal do Paraná (UFPR), município de Palotina. Os métodos utilizados foram sedimentação simples (Hoffmann, Pons e Janer) e flutuação em solução hipersaturada de cloreto de sódio (Willis-Mollay) ${ }^{7}$.

Os dados foram anotados em fichas separadas por recinto. Nos casos positivos, medidas de controle ambiental foram sugeridas para os recintos. Este trabalho foi avaliado e aprovado pela Comissão de Ética e Bem-estar Animal (CEBEA) do Campus Palotina da UFPR, protocolo n ${ }^{\circ}$ 29/2010.

\section{RESULTADOS E DISCUSSÕES}

Das 228 amostras de fezes analisadas 127 $(55,7 \%)$ foram positivas e destas, 55 (43,3\%) apresentaram infecção mista. Os parasitos encontrados pertencem aos gêneros: Strongyloides, Eimeria, Capillaria, Deletrocephalus e Isospora, às superfamílias Strongyloidea, Ascaroidea e Spiruroidea e à ordem Trichurida (Tabela 1, Figura 2). O único recinto que apresentou resultados negativos em todo o período avaliado foi o que abrigava as espécies Jabiru mycteia (tuiuiú) e Cariama cristata (seriema).

As amostras coletadas dos recintos de Pavo cristatus (pavão) e Rhea americana (araçari- poca) foram $100 \%$ positivas. Na sequência, as espécies que apresentaram um número maior de amostras positivas em relação às amostras coletadas foram Ara ararauna (arara vermelha) com $80 \%$ e Penelope ochrogaster (jacu) com 78,57\%. A espécie que apresentou maior prevalência de infecção mista foi Selenidera maculirostris (araçari-poca) (85,71\%), seguida pelo Pavo cristatus (pavão) com 78,57\%. 

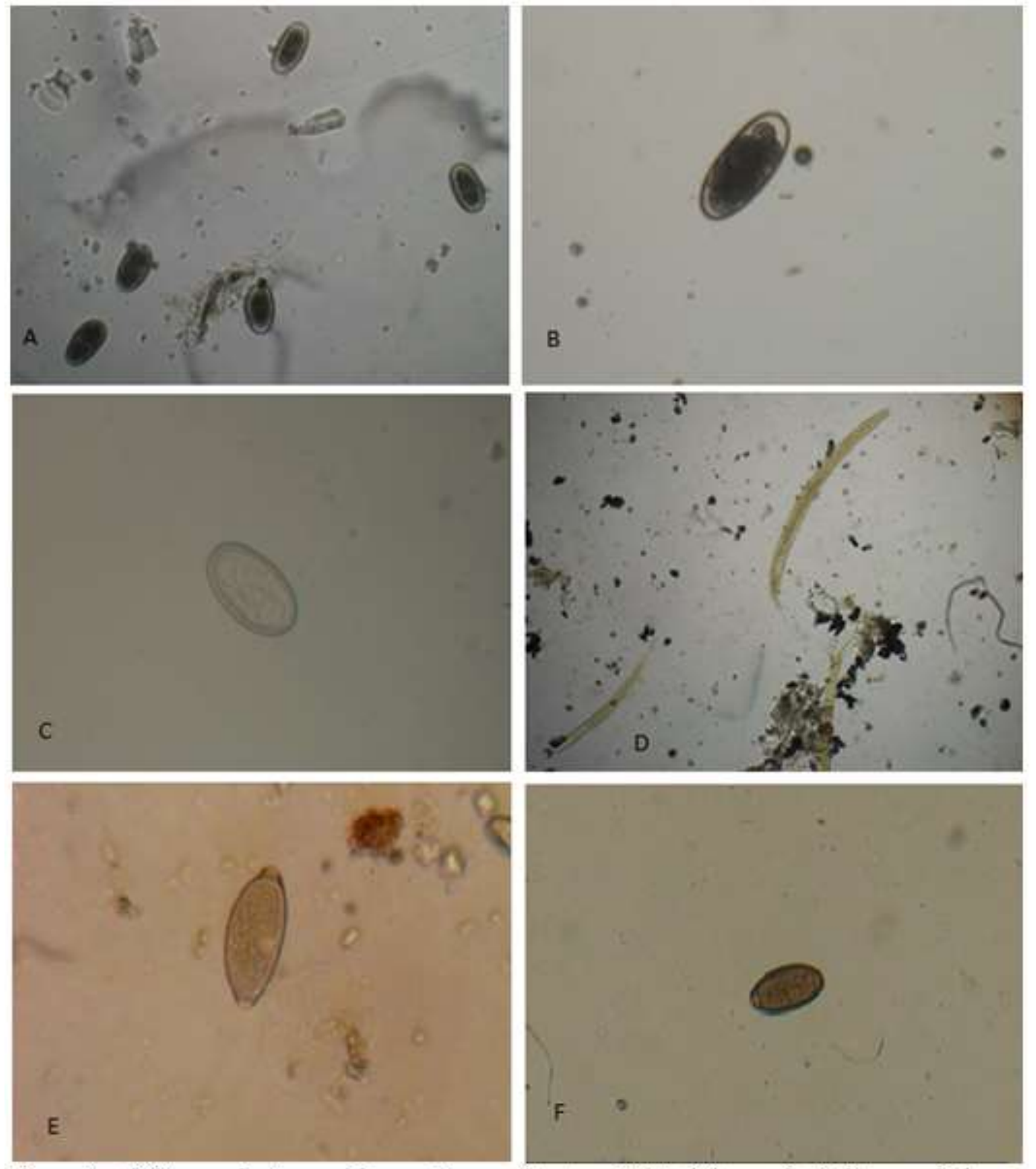

Figura 2 - (A) ovos de Ascaroidea em Pavo cristatus, 100X. (B) ovo de Deletrocephalus sp. em Rhea americana, 100X (C) ovo de Spiruroidea em Penelope ochrogaster, 400X. (D) Strongyloides sp. em Ara ararauna, 100X (E) ovo de Trichurida em Pavo cristatus, 400X. (F) ovo de Capillaria sp. em Penelope ochrogaster, 400X. 
Tabela 1. Resultados dos exames parasitológicos das aves cativas no Parque Municipal Danilo Galafassi em Cascavel-PR, Brasil

\begin{tabular}{|c|c|c|c|c|c|c|}
\hline Nome científico & Nome comum & Recintos & Amostras & Positivas & Mistas & Parasitos \\
\hline Pavo cristatus & Pavão & 1 & 14 & 14 & 11 & $\begin{array}{l}\text { Eimeria, Capillaria, Ascaroidea, } \\
\text { Spiruroidea, Trichurida }\end{array}$ \\
\hline $\begin{array}{l}\text { Jabiru mycterial } \\
\text { Cariama cristata }\end{array}$ & Tuiuiu/ Seriema & 1 & 13 & 0 & 0 & Negativo \\
\hline $\begin{array}{l}\text { Rhinoptynx } \\
\text { clamator }\end{array}$ & Coruja orelhuda & 1 & 10 & 2 & 0 & Strongyloides, Ascaroidea, \\
\hline Tyto alba & Coruja suindara & 1 & 13 & 2 & 1 & Strongyloides, Strongyloidea \\
\hline Rhea americana & Ema & 1 & 13 & 8 & 1 & Deletrocephalus, Strongyloidea \\
\hline $\begin{array}{l}\text { Penelope } \\
\text { ochrogaster }\end{array}$ & Jacus & 1 & 14 & 11 & 5 & $\begin{array}{l}\text { Strongyloides, Strongyloidea, Ascaroidea, } \\
\text { Spiruroidea, Capillaria, Eimeria }\end{array}$ \\
\hline & Coletivo aves & 1 & 8 & 5 & 1 & $\begin{array}{c}\text { Ascaroidea, Strongyloides, Strongyloidea } \\
\text { Eimeria }\end{array}$ \\
\hline $\begin{array}{l}\text { Mivalgo } \\
\text { chimachima }\end{array}$ & $\begin{array}{l}\text { Gavião } \\
\text { carrapateiro }\end{array}$ & 1 & 10 & 5 & 2 & Strongyloides, Ascaroidea, Eimeria \\
\hline $\begin{array}{c}\text { Heterospizias } \\
\text { meridionalis }\end{array}$ & Gavião caboclo & 1 & 9 & 2 & 1 & Strongyloides, Ascaroidea \\
\hline $\begin{array}{l}\text { Polyborus } \\
\text { plancus }\end{array}$ & Carcará & 1 & 11 & 5 & 1 & Strongyloidea, Strongyloides, Eimeria \\
\hline Ara ararauna & Arara Canindé & 1 & 13 & 9 & 6 & $\begin{array}{c}\text { Strongyloidea, Strongyloides, Eimeria } \\
\text { Capillaria }\end{array}$ \\
\hline \multirow[t]{3}{*}{$\begin{array}{l}\text { Cyanoliseus } \\
\text { patagonus }\end{array}$} & $\begin{array}{c}\text { Ararinha da } \\
\text { patagônia }\end{array}$ & 1 & 11 & 4 & 2 & Strongyloides, Ascaroidea, Spiruroidea \\
\hline & Coletivo araras & 1 & 9 & 7 & 1 & Strongyloides, Strongyloidea, Capillaria \\
\hline & $\begin{array}{c}\text { Coletivo } \\
\text { periquitos }\end{array}$ & 2 & 15 & 10 & 4 & $\begin{array}{l}\text { Strongyloides, Cestoda, Eimeria, } \\
\text { Ascaroidea, Strongyloidea }\end{array}$ \\
\hline $\begin{array}{l}\text { Ramphastus } \\
\text { dicolorus }\end{array}$ & $\begin{array}{l}\text { Tucano bico } \\
\text { verde }\end{array}$ & 2 & 20 & 11 & 4 & $\begin{array}{c}\text { Strongyloidea, Strongyloides, Eimeria, } \\
\text { Ascaroidea }\end{array}$ \\
\hline Ciconia maguari & Maguari & 1 & 13 & 7 & 3 & $\begin{array}{c}\text { Strongyloides, Strongyloidea, Eimeria, } \\
\text { Ascaroidea }\end{array}$ \\
\hline $\begin{array}{l}\text { Baillonius } \\
\text { bailloni }\end{array}$ & Araçari poca & 1 & 3 & 3 & 2 & $\begin{array}{c}\text { Strongyloides, Strongyloidea, } \\
\text { Eimeria }\end{array}$ \\
\hline Ara chloroptera & Arara vermelha & 1 & 10 & 7 & 6 & $\begin{array}{c}\text { Strongyloidea, Strongyloides, Isospora, } \\
\text { Ascaroidea }\end{array}$ \\
\hline Amazona aestiva & Papagaios & 1 & 7 & 5 & 1 & Strongyloides, Eimeria, Strongyloidea \\
\hline $\begin{array}{l}\text { Pteroglossus } \\
\text { castanotis }\end{array}$ & $\begin{array}{l}\text { Araçari } \\
\text { castanho }\end{array}$ & 1 & 12 & 9 & 3 & Strongyloides, Strongyloidea, Ascaroidea \\
\hline TOTAL & & 22 & 228 & 127 & 55 & \\
\hline
\end{tabular}

Os parasitos que obtiveram maior frequência foram: Strongyloides sp. $(59,84 \%)$ e Superfamília Strongyloidea $(26,77 \%)$ e Eimeria $(20,47 \%)$. Os parasitos que apresentaram menor frequência foram: Isospora sp. $(0,78 \%)$ e Classe Cestoda $(1,75 \%)$, conforme Tabela 2.

No estudo conduzido por MariettoGonçalves et al. $^{8}$, foram analisadas 207 amostras fecais de 45 espécies de aves provenientes de atendimentos do Hospital Veterinário da Faculdade de Medicina Veterinária e Zootecnia da Universidade Estadual Paulista, Campus de Botucatu-SP. Destas, apenas 16 espécies apresentaram exames positivos, sendo observados ovos de tricurídeos, Ascaridia spp., Heterakis spp., cistos de Balantidium spp., Blastocystis spp., Entamoeba spp. e oocistos de coccídeos.
Tabela 2. Frequência de parasitos encontrados em 127 amostras positivas de fezes de aves cativas do Parque Municipal Danilo Galafasi (Cascavel-PR) no período de agosto/2010 a julho/2012

\begin{tabular}{lc}
\hline Parasitos & Frequência (\%) \\
\hline Strongyloides & 59,84 \\
Strongyloidea & 26,77 \\
Eimeria & 20,47 \\
Ascaroidea & 18,11 \\
Capillaria & 11,02 \\
Trichurida & 8,66 \\
Deletrocephalus & 5,51 \\
Spiruroidea & 2,36 \\
Cestoda & 1,57 \\
Isospora & 0,78 \\
\hline
\end{tabular}

No estudo de Freitas et al. ${ }^{5}$, das 685 aves em cativeiro do Estado de Pernambuco analisadas, 320 $(46,7 \%)$ estavam parasitados com Capillaria sp., 
Strongyloides sp., Ascaridia sp., Heterakis sp., Strongyloidea, Spiruroidea, Cestoda, Trematoda, Coccidia, dados que corroboram os resultados obtidos nesta pesquisa.

Vicente et al. ${ }^{9}$ realizaram um inventário de nematódeos presentes em aves no Brasil, no qual descreveram Deletrocephalus sp. em Rhea americana, Ascaroidea em Penelope sp., Ara arauna e Ara chloroptera e Capillaria sp. em Pavo sp. Esses resultados condizem com a presente pesquisa.

Freitas et al. ${ }^{10}$ examinaram amostras de fezes de 685 aves cativas no Criadouro Cientifico e Cultural Chaparral em Pernambuco, das quais 320 apresentavam-se infectadas por Capillaria sp., Strongyloides sp., Ascaridia sp., Heterakis sp., Strongyloidea, Spiruroidea, coccídios e parasitos da classe Cestoda. Todos esses resultados corroboram com os resultadas das análises desta pesquisa. Além desses parasitos, os pesquisadores encontraram os da classe Trematoda e o protozoário Entamoeba sp.

O tamanho do cativeiro, tipo de confinamento, presença de contactantes externos, tipo de alimentação e fonte de fornecimento de água, podem induzir ao estresse e favorecer a proliferação e disseminação das doenças parasitárias ${ }^{7}$. Aves recém-chegadas ao criatório e aquelas que têm acesso ao chão têm maior propensão a infecções parasitárias.

Estudos relacionados ao levantamento parasitológico de animais em cativeiro contribuem para o conhecimento da fauna parasitária e auxiliam a sanar dúvidas sobre as diferentes espécies de parasitos e principalmente auxiliam na promoção de bem-estar para os animais cativos ${ }^{11}$.

As técnicas utilizadas neste estudo foram eficazes para a detecção de parasitos de aves silvestres e, por meio da positividade dos achados parasitológicos, puderam ser instituídas medidas profiláticas. Como medidas de controle foram sugeridas a limpeza e desinfecção regulares dos recintos, tratamento dos animais, análises coproparasitológicas regulares, bem como evitar a entrada de animais sinantrópicos. Estes últimos são considerados a principal fonte de infecção, pois acabam invadindo os recintos em busca de alimentos levando agentes para os animais cativos.

\section{CONCLUSÃO}

As aves cativas no parque municipal Danilo Galafassi em Cascavel-PR apresentaram-se parasitadas por helmintos e protozoários gastrintestinais, sendo helmintos os mais frequentes, não havendo casos clínicos relacionados, durante o período do estudo.

O presente trabalho colabora com informações sobre o parasitismo das espécies pesquisadas, uma vez que existem poucos trabalhos nessa área referentes a aves silvestres.

\section{REFERÊNCIAS}

1. Geraghty V, Mooney J, Pike K. A study of parasitic infections in mammals and birds at the Dublin Zoological Garden. Veterinary Research Communications. 1982. 5: 343-348.

2. Costa IA, Coelho CD, Bueno C, Ferreira I, Freire RB. Ocorrência de parasitos gastrintestinais em aves silvestres no município de Seropédica, Rio de Janeiro, Brasil. Ciência Animal Brasileira. 2010. 11 (4): 914-922

3. Katagiri S, Oliveira-Sequeira TCG. Zoonoses causadas por parasitas intestinais de cães e o problema do diagnóstico. Arquivos do Instituto Biológico. 2007. 74(2): 175-184.

4. Ippen R, Kojojed V, Jira J. Toxoplasmosis in Zoo animals. Folia Parasitologica. 1981. 28:109-115.

5. Freitas MFL, Oliveira JB, Cavalcanti MDB, Leite AS, Magalhães VS, Oliveira RA, Sobrino AE. Parasitos gastrointestinales de aves silvestres em cautiverio em El estado de Pernambuco, Brasil. Parasitologia Latinoamericano. 2002. 57 (1-2): 50-54.

6. Portal Do Municipio De Cascavel. Parques. 2010. [cited 2012 abr 20]. Available from: $<$ http://www.cascavel.pr.gov.br/secretarias/semdec/sub_p agina.php?id=258>.

7. Muller GCK, Greinert JÁ, Silva Filho HH. Frequência de parasitas intestinais em felinos mantidos em zoológicos. Arquivo Brasileiro de Medicina Veterinária e Zootecnia. 2005. 57 (4): 559-561.

8. Marietto-Gonçalves GA, Martins TF, Lima ET De, Lopes RS, Filho RL. A. Prevalência de endoparasitas em amostras fecais de aves silvestres e exóticas examinadas no laboratório e ornitopatologia e no laboratório de enfermidades Parasitárias da FMVZ Unesp/Botucatu, SP. Ciência Animal Brasileira. 2009. 10 (1): 349-354.

9. Vicente JJ, Rodrigues HO, Gomes DC, Pinto RM. Nematoides no Brasil - Parte IV: Nematoides de Aves. Revista Brasileira de Zoologia. 1995.12 (1): 1 - 27.

10. Freitas MFL, Oliveira JB, Cavalcanti MDB, Leite AS, Magalhães VS, Oliveira RA, Sobrino E. Parasitologia Latinoamericana. 2002. 57: 50 - 54.

11. Figueiredo MAP, Santos ACG, Guerra RMSNC. Ectoparasitos de animais silvestres no Maranhão. Pesquisa Veterinária Brasileira. 2010. 11 (30): 988-990. 